\title{
To what extent is personality associated with time perspective?
}

\author{
Anna Muro ${ }^{1 *}$, Judit Castellà2, Cristina Sotoca ${ }^{2}$, Santiago Estaún ${ }^{2}$, Sergi Valero ${ }^{3}$ \& Montserrat Gomà-i-Freixanet $^{1}$ \\ 1 Department of Health and Clinical Psychology, Universitat Autònoma de Barcelona \\ 2 Department of Basic, Developmental and Educational Psychology, Universitat Autònoma de Barcelona. \\ 3 Department of Psychiatry, Hospital Universitari Vall d'Hebron, CIBERS AM. Universitat Autònoma de Barcelona.
}

\begin{abstract}
Título: ¿Hasta qué punto está relacionada la personalidad con la perspectiva temporal?

Resumen Estudios recientes han analizado los correlatos conductuales de la perspectiva temporal (PT), sugiriendo que esta diferencia individual influye en muchos comportamientos relacionados con la salud, tales como el consumo de tabaco y otras sustancias, la actividad física o la satisfacción vital. Se sugiere que una PT consistentemente sesgada hacia un determinado marco temporal está asociada con algunos trastornos psiquiátricos y también está mediada por determinados factores de personalidad. Sin embargo, son escasos los estudios que han explorado la relación entre personalidad y PT, y ninguno de ellos bajo una perspectiva psicobiológica. El objetivo de este estudio fue examinar la relación entre el Inventario de Perspectiva Zimbardo Time (ZTPI) y Zuckerman-Kuhlman Personality Questionnaire (ZKPQ) en una muestra de 196 estudiantes universitarios a través de un análisis de regresión múltiple. Los resultados mostraron que: el pasado negativo correlaciona positivamente con el Neuroticismo-Ansiedad y negativamente con la Actividad; el presente hedonista correlaciona positivamente con la Impulsividad- Búsqueda de Sensaciones y la Sociabilidad en un grado muy alto, y el futuro correlaciona positivamente también con el Neuroticismo-Ansiedad y la Actividad, y negativamente con la ImpulsividadBúsqueda de Sensaciones. Los resultados se discuten en términos de la definición de ambas escalas, sus relaciones y sus implicaciones en campos aplicados.
\end{abstract}

Palabras clave: Personalidad; perspectiva temporal; ZKPQ; ZTPI

\section{Introduction}

Time perspective (TP) is a psychological construct that is considered as a basic function for human development and evolution (Kruger, Reischl, \& Zimmerman, 2008; Suddendorf, 2006; Zimbardo \& Boyd, 1999). It is defined as a cognitive tendency of individuals to be timely oriented toward the past, the present or the future. TP acts as a cognitive frame that mediates situational responses and behavioral patterns through time and situations, suggesting that TP may be a manifestation of a dispositional style or an individualdifference variable (Zimbardo \& Boyd, 1999). Therefore, TP is considered as a relatively stable and independent characteristic, through which personal and social experiences are assigned to different time frames giving order, coherence and meaning to life events.

It is hypothesized that healthy individuals would be cognitively balanced among past, present and future frames, but when individuals overemphasize one of the three frames, a cognitive temporal "bias" would appear influencing decision making (Taber, 2013). This bias might become a dispositional style that may predict individuals' specific responses across situations. Furthermore, extreme patterns of temporal biases

* Dirección para correspondencia [Correspondence address]:

Anna Muro. Department of Health and Clinical Psychology. Universitat Autònoma de Barcelona, 08193 Bellaterra (Spain). website: http://grupsderecerca.uab.cat/zkpq/ E-mail: anna.muro@uab.cat
Abstract: Recent research has focused on behavioral correlates of temporal perspective (TP), suggesting that this individual difference has an influence on many health-related behaviors such as smoking and substance use, physical activity or life satisfaction. It is suggested that a consistently biased temporal orientation is associated with some psychiatric disorders and mediated by personality factors. However, few studies have explored the relationship between personality and TP from a psychobiological approach. The aim of the study was to examine the relationship between the Zimbardo Time Perspective Inventory (ZTPI) and the ZuckermanKuhlman Personality Questionnaire (ZKPQ) in a sample of 196 undergraduate students through a multiple regression analysis. Results showed that: Past-Negative correlated positively with Neuroticism-Anxiety and negatively with Activity; Present Hedonistic correlated positively with Impulsive-Sensation Seeking and Sociability in a very high degree; and Future correlated positively with Neuroticism-Anxiety and Activity, and negatively with Impulsive-Sensation Seeking. Results are discussed in terms of the definition of both scales, their relationships and their implications in applied fields.

Key words: Personality; time perspective; ZKPQ; ZTPI

might negatively affect health-related behaviors. For example, individuals who are strongly biased toward the present might not consider the future consequences of their acts by decreasing risk perception of behaviors such as smoking, substance use, or physical inactivity (e.g., Adams \& Nettle, 2009; Apostolidis, Fieulaine, Simonin, \& Rolland, 2006; Boyd \& Zimbardo, 2005; Daugherty \& Brase, 2010). The relevance of a biased TP in abnormal psychological functioning has also been suggested by several authors indicating that TP could be considered a core feature of some psychiatric disorders such as depression and suicidal ideation (Laghi, Baiocco, D’Alessio, \& Gurrieri, 2009; van Beek, Berghuis, Kerkhof, \& Beekman, 2011).

Zimbardo and Boyd (1999) described five temporal frames, i.e., Past-Negative, Present-Hedonistic, Future, PastPositive and Present-Fatalistic through theoretical conceptualization and factorial analysis and developed the Zimbardo Time Perspective Inventory (ZTPI). Taking into account that distortions on someone's time perspective may be mediated by other individual differences, several studies have analyzed the relationship between TP and personality. Most of these studies have been conducted under the Five Factor Model (FFM) of personality. The seminal study of Zimbardo and Boyd (1999) using the Big Five Questionnaire (BFQ; Caprara, Barbaranelli, Borgogni, and Perugini, 1993) found that Past-Negative was negatively correlated with Emotional Stability; Present-Hedonistic was positively associated with Energy and negatively with Conscientiousness (C); Future 
was positively associated with $\mathrm{C}$ and Energy; Past-Positive correlated positively only with Friendliness; and PresentFatalistic was negatively associated with Conscientiousness and Energy. In this same validation study, results showed that the Sensation Seeking Scale (SSS; Zuckerman, Eysenck, \& Eysenck, 1978) was strongly and positively associated with Present-Hedonistic; while a significant negative correlation was found with Future. Recently, several studies (Adams \& Nettle, 2009; Daugherty \& Brase, 2011; Dunkel \& Weber, 2010; van Beek et al., 2011; Zhang \& Howell, 2011) have also analyzed the relationship between the FFM and TP using different assessment instruments. All together, these correlational studies have shown that the most replicated finding and the strongest associations between TP and personality are: a) Future TP is positively associated with Conscientiousness; b) Past-Negative is positively associated with Neuroticism and negatively with Extraversion, and c) PresentHedonistic is positively correlated with Extraversion.

In the present research, we aim to assess normal personality by the use of the Alternative Five Factor Model (AFFM) which has some advantages over the aforementioned models. The AFFM model (Zuckerman, Kuhlman, Joireman, Teta, \& Kraft, 1993; Zuckerman, Kuhlman, Thornquist, \& Kiers, 1991) emerged from a series of factor analyses of scales that had already been widely used in human psychobiological research. The basic factors in this AFFM are measured by the Zuckerman-Kuhlman Personality Questionnaire (ZKPQ; Zuckerman et al., 1993). It assesses five personality factors: Neuroticism-Anxiety (N-Anx), Activity (Act), Sociability (Sy), Impulsive-Sensation Seeking (ImpSS) and Aggression-Hostility (Agg-Host). In this model, Activity and Sociability constitute two separate factors of Extraversion. This conceptual and empirical differentiation may be heuristic in the conceptualization of time perspective theory helping to explain previous results regarding Extraversion. Besides, the dimension of Neuroticism from the ZKPQ does not include impulsivity nor hostility traits as the FFM's NEO PI-R does (Costa \& McCrae, 1992), instead, specific scales for these two traits are included. The ZKPQ is a valid and reliable tool that offers optimal psychometric properties in Spanish samples (Gomà-i-Freixanet \& Valero, 2008) and is embedded in a biological perspective (Zuckerman, 2005). On an applied basis, in a multivariate analysis level, the AFFM could provide a more refined association with time orientation.

Our aim was to explore the relationship between personality and TP, and to what extent TP could be related to a broader psychobiological personality model, namely the Alternative Five Factor Model. This is the first time that TP and the AFFM of personality are analyzed together. According to the ZTPI and ZKPQ scale definitions, we enunciated the following hypotheses: 1) Past-Negative focusing on a negative and pessimistic view of the past, would show a positive relationship with N-Anx; 2) Present-Hedonistic scale reflecting a hedonistic and risk taking attitude toward life would show a positive relationship with Act, Sy and ImpSS;
3) Future scale, measuring planning for and achievement of future goals, would show a negative association with ImpSS and a positive one with Act; 4) Past-Positive scale focusing on a positive attitude toward the past, would probably be negatively related to N-Anx, and 5) Present-Fatalistic would probably be positively associated with $\mathrm{N}$-Anx.

\section{Method}

\section{Participants}

An initial sample of 202 volunteer undergraduate students from the Autonomous University of Barcelona participated in the study. Only those who fully completed the two questionnaires were included in the data analyses. Inattention and social desirability were also controlled through the Infrequency scale of the ZKPQ, and participants scoring $>4$ were excluded (Gomà-i-Freixanet, Valero, Puntí, \& Zuckerman, 2004). The final sample $(n=196)$ consisted of 109 men $(55.6 \%)$ and 86 women, ranging from 18 to 35 years $(M$ age $=19.98, S D=2.94)$. La suma d'homes i dones dóna 195 , no 196

\section{Instruments}

Zuckerman-Kublman Personality Questionnaire (ZKPQ; Zuckerman et al., 1993). To assess personality, the Spanish adaptation of the ZKPQ (Gomà-i-Freixanet et al., 2004) was administered. It consists of 99 dichotomous items covering five personality scales and an additional Infrequency scale (Infreq, 10 items) assessing subjects with careless or social desirability responding. This scale ensures that none of the basic traits is affected by this response-set bias. The five assessed personality factors are: Neuroticism-Anxiety, Activity, Sociability, Impulsive Sensation-Seeking, and AggressionHostility and are described as follows: 1) NeuroticismAnxiety (N-Anx, 19 items) describes frequent emotional upset, tension, worry, fearfulness, indecision, lack of selfconfidence, and sensitivity to criticism (i.e., "I frequently get emotionally upset", "I often worry about things that other people think are unimportant"). 2) Activity (Act, 17 items) describes the need for general activity and impatience or restlessness when there is nothing to do, as well as a preference for challenging and hard work, an active busy life, and a high energy level (i.e., "I like a challenging task much more than a routine one"). 3) Sociability (Sy, 17 items) describes the number of friends and the amount of time spent with them, outgoingness at parties and a preference for being with others (i.e., "At parties, I enjoy mingling with many people whether I already know them or not"); it also indicates an intolerance for social isolation and for engaging in solitary activities (i.e., "I would not mind being socially isolated in some place for some period of time"). 4) Impulsive Sensation-Seeking (ImpSS, 19 items) is a factor that describes lack of planning, the tendency to act impulsively without thinking (i.e., "I usually think about what I am going 
to do before doing it"), and the seeking of excitement, novel experiences, and the willingness to take risks for these types of experiences (i.e., "I would like the kind of life where one is on the move and travelling a lot, with lots of change and excitement"). And 5) Aggression-Hostility (Agg-Host, 17 items) reflects a readiness to express verbal aggression, rude, thoughtless or antisocial behavior, vengefulness and spitefulness, having a quick temper, and impatience with others (i.e., "When people disagree with me I cannot help getting into an argument with them", "It's natural for me to curse when I am mad"). This instrument has shown good psychometric properties, with adequate internal consistency alpha coefficients and test-retest reliabilities (Gomà-iFreixanet et al., 2004; Muro, Gomà-i-Freixanet, \& Adan, 2009). The factorial structure has also been replicated in Spanish samples, including general population (Gomà-iFreixanet, Valero, Muro, \& Albiol, 2008). The instrument has also demonstrated consensual validity between auto/hetero-reports (Gomà-i-Freixanet, Wismeijer, \& Valero, 2005) and good discriminant validity in clinical samples (Martínez et al., 2010; Pascual et al., 2007; Valero et al., 2012). The ZKPQ also provides normative data for the general population (Gomà-i-Freixanet \& Valero, 2008).

Zimbardo Time Perspective Inventory (ZTPI; Zimbardo \& Boyd, 1999). To assess time perspective, the Spanish version of the ZTPI (Díaz-Morales, 2006) was administered. This version contains 56 items which are assessed on a 5-point scale ranging from 1 (very uncharacteristic) to 5 (very characteristic). The ZTPI consists of five factors: Past-Negative, Present-Hedonistic, Future, Past-Positive and PresentFatalistic. 1) The Past-Negative scale (10 items): reflects a generally negative, aversive view of the past (i.e.: "Painful past experiences keep being replayed in my mind", "I've made mistakes in the past that I could undo"). 2) The Present-Hedonistic scale (15 items) reflects a hedonistic, enjoyment and pleasure centered risk-taking attitude toward time and life (i.e. "I take risks to put excitement in my life", "I make decisions on the spur of the moment"). 3) The $\boldsymbol{F u}$ ture scale (13 items) reflects a general orientation toward the achievement of future goals and is characterized by planning and organization (i.e. "I am able to resist temptations when I know that there is work to be done", "I believe that a person's day should be planned ahead each morning"). 4) The Past-Positive scale (9 items) reflects a warm, sentimental attitude toward the past (i.e. "It gives me pleasure to think about my past", "Happy memories of good times spring readily to mind"). 5) The Present-Fatalistic scale (9 items) measures a helpless and hopeless attitude toward the future and life, and a sense that the future is predestined and not influenced by present individual actions (i.e. "My life path is controlled by forces I cannot influence", "Since whatever will be will be, it doesn't really matter what I do"). The ZTPI has been translated into different languages (Carelli, Wiberg, \& Wiberg, 2011; Milfont, Andrade, Pessoa, \& Belo, 2008; Sirkova \& Mitina, 2008), including Spanish (Díaz-Morales, 2006).

\section{Procedure}

The questionnaires were administered by the researchers and participants completed the questionnaires anonymously in classrooms settings. All participants gave informed consent prior to their inclusion in the study and did not receive any credit or economic reward for their collaboration. The Ethical Committee of the university approved the protocol.

\section{Data Analyses}

Data analyses followed two steps. In the first step, we calculated means, standard deviations and Cronbach's alphas. We tested mean differences between sex groups by means of a two-tailed independent Student's $t$-test. To identify significant associations between ZKPQ and ZTPI scales, we calculated Pearson's correlation coefficients. On a second step, in order to ascertain which ZKPQ personality factors would be the most associated with ZTPI scales, we performed a multiple regression analysis. Data analyses were performed using the SPSS statistical package (version 20.0), and statistical tests were bilateral with Type I error set at $5 \%$.

\section{Results}

\section{Descriptive statistics}

Means, standard deviations and internal consistencies (Cronbach's $\alpha$ ) of the ZKPQ and the ZTPI scales for the total sample are shown in Table 1 . No significant differences were found between sex groups on any of the ZKPQ and ZTPI scales, except Agg-Host $(t=2.76, p=0.006)$. Cronbach's $\alpha$ of the ZKPQ personality scales ranged from .71 to .88 , with a mean of .80 . Cronbach's $\alpha$ of the first three ZTPI scales ranged from .74 to .83 . Present-Fatalistic $(\alpha=$ .33) and Past-Positive $(\alpha=.63)$ showed a low internal consistency, accordingly, these two scales were not included in further analyses.

Table 1. Means, standard deviations and Cronbach's $\alpha$ for ZKPQ and ZTPI scales.

\begin{tabular}{lccc}
\hline ZKPQ & $M$ & $S D$ & Cronbach's $a$ \\
\hline N-Anx & 9.86 & 5.09 & .88 \\
Act & 7.99 & 3.59 & .76 \\
Sy & 8.45 & 3.85 & .81 \\
ImpSS & 10.08 & 4.29 & .82 \\
Agg-Host & 8.23 & 3.16 & .71 \\
Infreq & 0.99 & 1.25 & \\
ZTPI & & & \\
\hline Past-Negative & 2.89 & 0.77 & .83 \\
Present-Hedonistic & 3.41 & 0.57 & .83 \\
Future & 3.38 & 0.57 & .74 \\
Past-Positive & 3.62 & 0.52 & .63 \\
Present-Fatalistic & 2.68 & 0.57 & .33 \\
\hline Notes ZKPQ Zuck
\end{tabular}

Notes: ZKPQ $=$ Zuckerman-Kuhlman Personality Questionnaire; N-Anx = Neuroticism-Anxiety; Act = Activity; Sy = Sociability; ImpSS = Impulsive Sensation Seeking; Agg-Host = Aggression-Hostility; Infreq = Infrequency; ZTPI = Zimbardo Time Perspective Inventory. 
Table 2 shows Pearson's correlations among scales of the ZKPQ and ZTPI. Past-Negative correlated positively and significantly only with $\mathrm{N}$-Anx $(r=.60)$. Present-Hedonistic correlated positively and significantly with all the ZKPQ scales, except N-Anx. It is worth mentioning the high correlation found between Present-Hedonistic and ImpSS $(r=$ $.74)$ and $\mathrm{Sy}(r=.54)$. Future correlated significantly and negatively with ImpSS $(r=-.48)$ and positively with $\mathrm{N}-\mathrm{Anx}(r=$ $.29)$.

Table 2. Pearson's correlations between ZKPQ and ZTPI scales.

\begin{tabular}{lccccc}
\hline \multirow{2}{*}{ ZTPI } & \multicolumn{5}{c}{ ZKPQ } \\
\cline { 2 - 6 } Past- & N-Anx & Act & Sy & ImpSS & Agg-Host \\
Negative & $.597^{* *}$ & -.049 & -.135 & -.039 & .098 \\
$\begin{array}{l}\text { Present- } \\
\text { Hedonistic }\end{array}$ & -.090 & $.298^{* *}$ & $.539^{* *}$ & $.736^{* *}$ & $.205^{* *}$ \\
Future & $.285^{* *}$ & .171 & -.091 & $-.478^{* *}$ & -.081 \\
\hline
\end{tabular}

Notes: ZKPQ $=$ Zuckerman Kuhlman Personality Questionnaire; $\mathrm{N}$-Anx $=$ Neuroticism-Anxiety; Act $=$ Activity; $S_{y}=$ Sociability; ImpSS = Impulsive Sensation Seeking; Agg-Host = Aggression-Hostility; ZTPI = Zimbardo Temporal Perspective Inventory.

$* * p<.01$, two-tailed.

\section{Multiple regression analyses}

A multiple regression analysis was executed for each of the three ZTPI scales that showed adequate internal consistency: Past-Negative, Present-Hedonistic and Future. The ZKPQ scales were considered as independent variables and a stepwise procedure was carried out to select significant variables. Results of the three analyses are summarized in Table 3.

Table 3. Multiple Regression Analyses ZTPI outputs of ZKPQ scales.

\begin{tabular}{llllll}
\hline & Predictors & Beta & $t$ & Sig. & $\mathrm{R}^{2}$ \\
\hline Past-Negative & N-Anx & .602 & 10.30 & .001 & \\
& Act & -.134 & 2.30 & .023 & .359 \\
\hline Present-Hedonistic & ImpSS & .643 & 13.64 & .001 & \\
& Sy & .284 & 6.03 & .001 & .629 \\
\hline Future & ImpSS & -.594 & 9.49 & .001 & \\
& Act & .336 & 5.35 & .001 & \\
& N-Anx & .184 & 3.17 & .002 & .386 \\
\hline
\end{tabular}

Notes: N-Anx = Neuroticism-Anxiety; Act = Activity; ImpSS = Impulsive Sensation Seeking; Sy = Sociability.

In the case of the Past-Negative variable, the significant personality factors associated were $\mathrm{N}-\operatorname{Anx}(\beta=.60 ; t=$ $10.30 ; p<.001)$ and Act $(\beta=-.13 ; t=2.30 ; p=0.023)$. Both variables accounted for $36 \%$ of the total variance of the Past-Negative factor. For the Present-Hedonistic factor, the significant variables were ImpSS $(\beta=.64 ; t=13.64 ; p<.001)$ and $\mathrm{Sy}(\beta=.28 ; t=6.03 ; p<.001)$. While correlations showed a significant association between Present-Hedonistic and Act, at a multivariable level this relationship did not hold after entering all personality variables in the regression analysis. The resulting total variance explained was $63 \%$. Finally, Future scale was significantly associated with ImpSS $(\beta=$ $.59 ; t=9.49 ; p<.001)$, Act $(\beta=.34 ; t=5.35 ; p<.001)$ and
N-Anx $(\beta=.18 ; t=3.17 ; p=0.002)$. These three personality variables explained $39 \%$ of the total variance of the Future scale.

\section{Discussion and conclusions}

The goal of the present study was to assess, for the first time, the relationship between personality and time perspective using the ZKPQ and the ZTPI. Regarding the reliability of these two assessment instruments, the magnitude of the $\alpha$ coefficients of the ZKPQ in this sample was adequate and similar to the coefficient estimates obtained in previous studies with Spanish student samples (Gomà-i-Freixanet et al., 2004; Muro et al., 2009), indicating a good internal consistency for all the ZKPQ scales. However, only three of the five ZTPI scales obtained adequate $\alpha$ coefficients of internal consistency, while low to medium reliabilities for PresentFatalistic and Past-Positive scales respectively were obtained. According to Crokett, Weinman, Hankins, and Marteu (2009), a possible explanation of the low reliability of these two aforementioned scales could be the inclusion of items that do not measure specifically TP, but do refer to other aspects of behavior (Worrell, Mello, \& Buhl, 2013).

Regarding the association between ZKPQ and ZTPI scales, only three of the five hypotheses could be tested due to the reliability problems of the two aforementioned ZTPI scales. Therefore, from the statistical point of view only correlations higher or equal to .30 were considered, and thus the first and second hypotheses were fully confirmed while the third hypothesis was only partially confirmed. It is worth mentioning that the strong correlation found between Present-Hedonistic and ImpSS, may suggest that there might be an overlap between both constructs. It should be noted that the similarity in content between the items of the PresentHedonistic scale and those of the ImpSS scale could be due to the fact that the Sensation Seeking Scale emphasizes present oriented functioning (Zuckerman, 1994) and that some of the items were taken from the SSS (Zuckerman et al., $1978)$ to construct the original Present-Hedonistic scale (Zimbardo \& Boyd, 1999).

Results regarding the association between TP and personality are similar to those obtained in other studies. PastNegative was strongly associated with N-Anx, either measured by the NEO PI-R (van Beek et al., 2011), the BFQ (Zimbardo \& Boyd, 1999) or the BFI (e.g., Dunkel \& Weber, 2010). Present-Hedonistic was strongly associated with ImpSS and Sy, and to a lesser extent with Act. Regarding Sy and Act, two of the main traits of Extraversion (E), van Beek et al. (2011) found that Present-Hedonistic correlated with E, and Zimbardo and Boyd (1999) using the Energy scale of the BFQ also found this same result. In relation to the results regarding the ImpSS scale of the ZKPQ, Zimbardo and Boyd (1999) also found that Present-Hedonistic was positively related to the SSS scale and negatively to the Conscientiousness domain of the BFQ. These results are in accordance to those obtained in our study in that the SS items 
from the ImpSS scale were extracted from the original version of the SSS. Regarding the Future scale, several studies have systematically obtained a positive correlation with $\mathrm{C}$ (e.g., Adams \& Nettle, 2009; van Beek et al., 2011) and the study of Zimbardo and Boyd (1999) a negative one with SSS. Taking into account the expected pattern of correlations between the ZKPQ and the NEO PI-R (Zuckerman et al., 1993) results obtained in relation to the Future scale are in the predicted direction.

Our second goal was to assess, at a multivariate level, to what extent the AFFM of personality would be associated with time perspective frames. Results obtained from the regression analyses indicate that Past-Negative was mostly associated with high $\mathrm{N}$-Anx scores, together with low Act levels. These results suggest that the cognitive tendency of focusing in past negative experiences might be highly developed in individuals with high anxiety levels, alertness and cautious behaviors. Being aware of past negative events might help to warn and prevent from foreseen dangers and threats. Low levels on Act also suggest that individuals who do not have energy for an active, busy life or without challenges to be actively attained, might develop a bias on focusing in past negative experiences that in turn might contribute to increase their anxiety levels (Boyd \& Zimbardo, 2005). ImpSS and Sy together were associated with a higher Present-Hedonistic cognitive tendency, suggesting that individuals who behave through a non-planned life-style with a preference for seeking excitement and novel experiences, and enjoying being with friends rather than engaging in solitary activities are mentally oriented toward present enjoyment, immediate pleasure and excitement without sacrifices today for rewards tomorrow (Zimbardo \& Boyd, 1999). Accordingly, ImpSS and Sy are associated with a temporal frame reflecting a hedonistic attitude toward time and life not taking into account future consequences (Boyd \& Zimbardo, 2005; Strathman, Gleicher, Boninger, \& Edwards, 1994). Finally, Future was associated with low ImpSS, high Act and to a lesser extent with high N-Anx scores. Therefore, and according to previous studies on behaviors associated with future consequences (Adams \& Nettle, 2009; Daugherty \& Brase, 2010; Zhang \& Howell, 2011), the future frame is associated with Conscientiousness facilitating individuals the control of immediate impulses and the adjustment of planned responses that regulate present behaviors that might cause future losses (Strathman et al., 1994; Zimbardo \& Boyd, 2009). Additionally, a cognitive regulation of expectancies to achieve future oriented goals might involve a need of investing energy and increasing activity levels (Boyd \& Zimbardo, 2005). However, N-Anx also appears to be associated with future TP. Accordingly, the association between N-Anx and Future

\section{References}

Adams, J., \& Nettle, D. (2009). Time perspective, personality and smoking, body mass, and physical activity: An empirical study. British Journal of Health Psychology, 14, 83-105.
TP reinforces the idea that future oriented individuals tend to increase their attention and alertness toward signals of future dangers to regulate behaviors in order to avoid negative consequences in the future.

It is also worth noting that Act and Sy, two of the main traits of Extraversion (Eysenck, 1967; Zuckerman et al., 1993) were associated with different TP frames; the former with Past-Negative and Future, and the latter with PresentHedonistic. These results reinforce the notion that both Act and Sy traits are independent with different behavioral pathways. Support for separating Act and Sy traits, however, is found when examining the beta weights in Table 3; these two traits are uniquely associated with different TP frames suggesting that they have divergent predictive validity.

This study has several limitations which limit the generalizability of the obtained data. The sample was composed by students with a short age range and a narrow socioeconomic and cultural representation thus questioning the representativeness of the general population. Furthermore, the low internal consistencies of Past-Positive and Present-Fatalistic scales merit a revision of the Spanish adaptation of the ZTPI. Recent findings show that individual differences in TP are important psychological variables related to a large array of variables such as life satisfaction (Zhang \& Howell, 2011), healthy behaviors (Daugherty \& Brase, 2011) and psychiatric problems (van Beek et al., 2011). On an applied basis for cognitive therapies, on the one hand, it would be worth reframing situations by altering one's time perspective frame to be more past positive, less past negative, and more pleasure oriented in the present and future, specially on anxiety, depression or stress related disorders. On the other hand, it would be worth reframing time perspective of those impulsive-sensation seekers and highly sociable subjects with vulnerability to suffer from health risky or impulsive behaviors, by balancing future and present hedonistic perspectives. This cognitive temporal modification would allow them to selfregulate and improve their decision making and to decrease non-adaptative impulsive behaviors that might lead them to negative future consequences such as alcoholism, substance abuse or lower academic achievement (Boyd \& Zimbardo, 2005).

The implications of this study highlight the importance of how individual differences in personality might predict temporal cognitive frames. The use of psychobiological models of personality (Eysenck, 1967; Zuckerman, 2005) in the study of TP is also encouraged since they might provide a better understanding of the behavioral evolutionary roots which involve cognitive regulation, and a sense of continuity and coherence needed for a successful adaptation.

Apostolidis, T., Fieulaine, N., Simonin, L., \& Rolland, G. (2006). Cannabis use, time perspective and risk perception: Evidence of a moderating effect. Psychology and Health, 21, 571-592. 
Boyd, J. N., \& Zimbardo, P. G. (2005). Time Perspective, Health, and Risk Taking, in A. Strathman \& J. Joireman (Eds.), Understanding Behavior in the Context of Time: Theory, Research, and Application (pp.85-107). New Jersey: Lawrence Erlbaum Associates, Inc.

Caprara, G. V., Barbaranelli, C., Borgogni, L., \& Perugini, M. (1993). The Big Five Questionnaire: A new questionnaire to assess the five factor model. Personality and Individual Differences, 15, 281-288.

Carelli, M. G., Wiberg, B., \& Wiberg, M. (2011). Development and Construct Validation of the Swedish Zimbardo Time Perspective Inventory. European Journal of Psychological Assessment, 27, 220-227

Costa, P. T., \& McCrae, R. R. (1992). NEO PI-R professional manual. Odessa, FL: Psychological Assessment Resources, Inc.

Crockett, R., Weinman, J., Hankins, M., \& Marteau, T. M. (2009). Time orientation and health-related behaviour: measurement in general population samples. Psychology and Health, 24, 333-350.

Daugherty, J. R., \& Brase, G. L. (2010). Taking time to be healthy: Predicting health behaviors with delay discounting and time perspective. Personality and Individual differences, 48, 202-207.

Díaz-Morales, J. F. (2006). Estructura factorial y fiabilidad del Inventario de Perspectiva Temporal de Zimbardo. Psicothema, 18, 565-571.

Dunkel, C. S., \& Weber, J. L. (2010). Using three levels of personality to predict time perspective. Current Psychology, 29, 95-103.

Eysenck, H. J. (1967). The biological basis of personality. Springfield, IL: Thomas.

Gomà-i-Freixanet, M., \& Valero, S. (2008). Spanish normative data of the Zuckerman-Kuhlman Personality Questionnaire (ZKPQ) in a general population sample. Psicothema, 20, 324-330.

Gomà-i-Freixanet, M., Valero, S., Muro, A., \& Albiol, S. (2008). Zuckerman-Kuhlman Personality Questionnaire: Psychometric properties in a sample of the general population. Psychological Reports, 103, 845-856.

Gomà-i-Freixanet, M., Valero, S., Puntí, J., \& Zuckerman, M. (2004). Psychometric properties of the Zuckerman-Kuhlman personality questionnaire in a Spanish sample. European Journal of Psychological Assessment, 20, 134-146.

Gomà-i-Freixanet, M., Wismeijer, A. A. J., \& Valero, S. (2005). Consensual Validity Parameters of the Zuckerman-Kuhlman Personality Questionnaire: Evidence From Self-Reports and Spouse Reports. Journal of Personality Assessment, 84, 279-286.

Kruger, D. J., Reischl, T., \& Zimmerman, M. A. (2008). Time Perspective as a mechanism for functional developmental adaptation. Journal of Social, Evolutionary, and Cultural Psychology, 2, 1-22.

Laghi, F., Baiocco, R., D’Alessio, M., \& Gurrieri, G. (2009). Suicidal ideation and time perspective in high school students. European Psychiatry, 24, 41-46.

Martínez, Y., Bosch, R., Gomà-i-Freixanet, M., Valero, S., Ramos-Quiroga, J. A., Nogueira, M., \& Casas, M. (2010). Variables diferenciales de personalidad en los subtipos de TDAH en la edad adulta. Psicothema, 22, 236-241.

Milfont, T. L., Andrade, P. R., Pessoa, V. S., \& Belo, R. P. (2008). Testing Zimbardo Time Perspective Inventory in a Brazilian sample. Interamerican Journal of Psychology, 42, 49-58.

Muro, A., Gomà-i-Freixanet, M., \& Adan, A. (2009). Morningnesseveningness, gender, and the Alternative Five Factor Model of Personality. Chronobiology International, 26, 1235-1248.
Pascual, J. C., Soler, J., Baiget, M., Cortés, A., Menoyo, A., Barrachina, J., ... Pérez, V. (2007). Association between the serotonin transporter gene and personality traits in borderline personality disorder patients evaluated with Zuckerman-Zuhlman Personality Questionnaire (ZKPQ). Actas Españolas de Psiquatría, 35, 382-386.

Sircova, A., \& Mitina, O. V. (2008). Zimbardo Time Perspective Inventory: A comparison across cultures [Abstract]. International Journal of Psychology, $3 / 4,43$.

Strathman, A., Gleicher, F., Boninger, D. S., \& Edwards, C.S. (1994). The consideration of future consequences: Weighing immediate and distant outcomes of behavior. Journal of Personality and Social Psychology, 66, 742752 .

Suddendorf, T. (2006). Foresight and evolution of the human mind. Science, 312, 1006-1007.

Taber, B. J. (2013). Time Perspective and Career Decision-Making Difficulties in Adults. Journal of Career Assessment, 21, 200-209. doi: $10.1177 / 1069072712466722$

Valero, S., Ramos-Quiroga, A., Gomà-i-Freixanet, M., Bosch, R., GómezBarros, N. Nogueira, M., ... Casas, M. (2012). Personality profile of adult ADHD: The alternative five factor model. Psychiatry Research, 198(1), 130-134.

Van Beek, W., Berghuis, H., Kerkhof, A., \& Beekman, A. (2011). Time Perspective, Personality and Psychopathology: Zimbardo's Time Perspective Inventory in Psychiatry. Time \& Society 20, 364-374.

Worrell, F. C., Mello, Z. R., \& Buhl, M. (2013). Introducing English and German versions of the Adolescent Time Attitude Scale (ATAS). Assessment, 20, 496-510.. doi:10.1177/1073191110396202

Zhang, J., \& Howell, R. T. (2011). Do time perspectives predict unique variance in life satisfaction beyond personality traits? Personality and Individual Differences, 50,1261 - 1266

Zimbardo, P. G., \& Boyd, J. N. (1999). Putting time in perspective: A valid, reliable individual differences metric. Journal of Personality and Social Psychology, 77, 1271-1288.

Zimbardo, P., \& Boyd, J. (2009). The Time Paradox: The new psychology of Time that will change your life. Thorndike press: USA.

Zuckerman, M. (1994). Behavioural Expressions and Biosocial Bases of SensationSeeking. Cambridge: Cambridge University Press.

Zuckerman, M. (2005). Psychobiology of personality (2nd Edition). Cambridge: Cambridge University Press.

Zuckerman, M., Eysenck, S. B. G., \& Eysenck, H. J. (1978). Sensation Seeking in England and America: Cross-cultural, age, and sex comparisons. Journal of Consulting and Clinical Psychology, 46, 139-149.

Zuckerman, M., Kuhlman, D. M., Joireman, J., Teta, P., \& Kraft, M. (1993). A comparison of three structural models for personality: The big three, the big five and the alternative five. Journal of Personality and Social Psycho$\log y, 65,757-768$.

Zuckerman, M., Kuhlman, D. M., Thornquist, M., \& Kiers, H. (1991). Five (or three): Robust questionnaire scale factors of personality without culture. Personality and Individual Differences, 12, 929-941.

(Article received: 04-04-2013; revised: 04-06-2013; accepted: 14-02-2014) 